\title{
Verschlechtern NSAR den Effekt der antidepressiven Pharmakotherapie?
}

Hintergrund: Nicht-steroidale antiinflammatorische Pharmaka (NSAR) und Antidepressiva sind zwei der verbreitetsten Klassen von Medikamenten, weshalb negative Effekte der Kombination beider von weitgehender Bedeutung wären. Hinweise auf negative Effekte der Kombination von selektiven SerotoninWiederaufnahmehemmern (SSRI) und NSAR ergaben sich zwar aus Tierversuchen und aus Daten $\operatorname{der} \operatorname{STAR}^{\star} \mathrm{D}$-Studie [1], allerdings muss der Einfluss weiterer Variablen dabei bedacht werden. Deshalb wurden in der vorliegenden Studie einerseits die $\mathrm{STAR}^{*} \mathrm{D}$-Daten reanalysiert und andererseits eine Kohorte therapieresistenter depressiver Patienten im Hinblick auf NSAREffekte untersucht.

Patienten und Methodik: Im ersten Teil der Studie wurden nahezu 130.000 Patienten mit der ICD-9-Diagnose einer Depression in Hinblick auf den dokumentierten Therapieerfolg mit einerseits einer Monotherapie mit SSRI, andererseits der Kombination von NSAR und einem Antidepressivum untersucht. In einem zweiten Schritt wurden die NSAR weiter differenziert in „klassische“ NSAR, Cyclooxygenase-2-(COX-2-) Inhibitoren und Salicylate. Schließlich wurde noch die Dauer der NSARVerschreibungen berücksichtigt. Aus den Daten der STAR ${ }^{\star} \mathrm{D}$ Studie wurden zunächst die Patienten, die Citalopram erhalten hatten, auf die Remission unter kombinierter Verschreibung mit NSAR hin untersucht, in einem zweiten Schritt die CitalopramNonresponder auf die Effekte von kognitiver Verhaltenstherapie (KVT). Die Effekte interferierender Variablen wurden dabei mit berücksichtigt.

Ergebnisse: Die Grobanalyse ergab zunächst, dass die NSAREinnahme mit Behandlungsresistenz assoziiert war. Allerdings zeigte sich dies nicht in den Gruppen der COX-2-Inhibitoren und der Salicylate, sondern spezifisch war der chronische Gebrauch von „klassischen“ NSAR mit Therapieresistenz verbunden. Dies war auch ein Ergebnis der STAR ${ }^{\star} D$-Analyse. Interessanterweise war darüber hinaus der Gebrauch von Narkotika, denen ein völlig anderer molekularer Mechanismus als den NSAR zugrunde liegt, mit einem schlechteren Outcome assoziiert. Schließlich respondierten Patienten mit NSAR-Gebrauch auch schlechter auf KVT. Patienten mit NSAR-Einnahme hatten andererseits signifikant mehr neurologische und muskelskelettale Probleme.

Gallagher PJ, Castro V, Fava M et al. Antidepressant response in patients with major depression exposed to NSAIDs: A pharmacovigilance study. Am J Psychiatry 2012; 169: 1065-72
Schlussfolgerungen: Die Autoren folgern, dass das schlechtere Outcome der antidepressiven Therapie unter der Kombination von NSAR und Antidepressiva nicht auf pharmakologische oder molekulare Interaktionen der beiden Substanzgruppen zurückzuführen ist, sondern vielmehr eher auf die bestehenden Komorbiditäten, weshalb die Verordnungen erfolgen. Vor allem bei chronischen Schmerzsyndromen ist der Behandlungserfolg von Antidepressiva, aber auch von KVT gering.

\section{-Kommentar von Norbert Müller, München}

\section{NSAR-Patienten sind meist kränker}

Auf der Analyse von Verordnungen basierende Pharmakovigilanzstudien sind per se methodisch anfällig. Die Studie geht nicht über die Evidenzstufe 3 hinaus. Allerdings haben die Autoren dieser Studie die Ergebnisse intensiv auf interferierende Variablen untersucht. Mit der Einnahme von „klassischen“ NSAR in Kombination mit Antidepressiva ebenso wie mit KVT waren mehr Therapieresistenz und ein schlechteres Outcome verbunden, allerdings nicht mit NSAR-artigen Substanzen wie selektiven COX-2-Inhibitoren oder Salicylaten. Das schlechte Ansprechen auf die Behandlung lässt sich am besten mit den Komorbiditäten dieser Patientengruppe erklären. Daraus zu folgern, COX-2-Inhibitoren und Salicylate die bei dieser Patientengruppe nicht mit einem schlechteren Ergebnis assoziiert sind - hätten eine zusätzliche unterstützende antidepressive Wirkung, würde bei der Interpretation dieser Daten zu weit gehen. Allerdings haben doppelblinde, randomisierte Studien mit COX-2-Inhibitoren gerade dies, nämlich zusätzliche antidepressive Effekte (bei Patienten ohne Komorbiditäten), gezeigt [2].
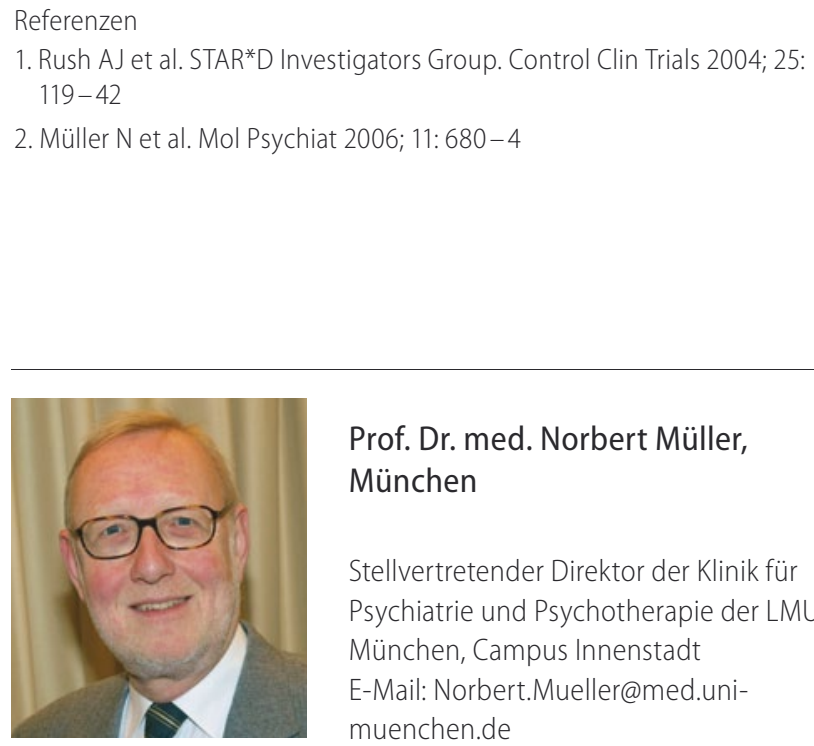

Prof. Dr. med. Norbert Müller, München

Stellvertretender Direktor der Klinik für Psychiatrie und Psychotherapie der LMU München, Campus Innenstadt E-Mail: Norbert.Mueller@med.unimuenchen.de 